\title{
Phenomenology of forward hadrons in deep inelastic scattering: Fracture functions and its $Q^{2}$ evolution
}

\author{
D. de Florian* \\ Theoretical Physics Division, CERN, CH 1211, Geneva 23, Switzerland \\ R. Sassot \\ Departamento de Física, Universidad de Buenos Aires, Ciudad Universitaria, Pabellón.1 (1428) Buenos Aires, Argentina
}

(Received 13 January 1997)

\begin{abstract}
We analyze recent data on the production of forward neutrons in deep inelastic scattering at DESY HERA in the framework of a perturbative QCD description for semi-inclusive processes, which includes fracture functions. Using a model estimate for the nonperturbative piece of the fragmentation process, in fairly good agreement with the available data, we analyze the $Q^{2}$ dependence of the resulting fracture functions, which is driven by nonhomogeneous evolution equations. We also propose a measurement of the pion production cross section in the target fragmentation region as a new test of perturbative QCD, which in this case predicts also a different $Q^{2}$ evolution with respect to the one given by the usual Altarelli-Parisi equations.

[S0556-2821(97)04711-5]

PACS number(s): 13.87.Fh, 13.85.Ni
\end{abstract}

\section{INTRODUCTION}

During the last three decades, deep inelastic scattering (DIS) has shown to be the most efficient experiment designed to extract information about the hadron structure. With the advent of new and more powerful accelerators, it has been possible to make new tests of QCD in a hitherto forbidden kinematical region $\left(x \ll 1\right.$ and $\left.Q^{2} \gg M_{p}^{2}\right)$, obtaining very interesting results on the behavior of the structure function $F_{2}$, in perfect agreement with the energy scale evolution predicted by the next to leading order (NLO) Altarelli-Parisi (AP) [1] equations and its approximation in the double asymptotic limit [2].

Moreover, with analyses of the longitudinal structure function $F_{L}$ and the above-mentioned scaling violations, it has been possible to constrain, if not to measure, the gluon distribution over a large kinematical range and also to obtain reliable results for the strong coupling constant $\alpha_{s}$. The use of evolution equations has not been limited to unpolarized scattering and its role has been preponderant in understanding the gluonic contribution to the Ellis-Jaffe spin-dependent sum rules [3] through the U(1) anomaly [4] and, therefore, in clarifying the situation with respect to the so-called "spin crisis." In that way, a large program has been developed around the measurement and the theoretical analysis of DIS structure functions with successful results on both sides, making DIS experiments one of the most important tests of QCD.

Also, within DIS experiments, other interesting processes are now under study at the DESY ep collider HERA, specifically events of a diffractive nature or, more precisely, with large rapidity gaps [5]. For them, the proton remains almost intact after interaction with the photon, changing only slightly its momentum and leaving the interaction region in

*Electronic address: Daniel.de.Florian@cern.ch the very forward direction without being detected. This particular kind of process has been recently tested by the ZEUS Collaboration [6], which has included a Leading Proton Spectometer in order to detect hadrons produced in the forward direction, and has measured the dependence of the diffractive cross section on the transferred momentum $(t)$ between the initial and the final protons. In this, they have obtained results compatible with previous determinations, all of them in good agreement with the effective Pomeron exchange picture, where the cross section is factorized into the product of a flux of Pomerons in the proton $\left(\propto x_{\mathrm{P}}^{-a}\right)$ times the, expectedly universal, distribution of partons in the Pomeron.

More recently, the ZEUS Collaboration [7] has measured events where neutrons, instead of protons, are produced in the forward direction, obtaining a sizable contribution of leading neutrons to the DIS cross section (as much as $10 \%$ ). This opens a new window to study hard processes in a new kinematical region hitherto considered only in soft analyses.

Along with these experimental improvements, a new theoretical idea has been developed in order to deal with such kind of forward processes. In a more general perturbative picture of semi-inclusive processes, it has been shown [8] that both current and the target fragmentation regions have to be considered: the first one by means of a cross section that can be factorized into a hard cross section, parton distributions, and fragmentation functions and in the second one, with another perturbatively computable hard cross section but introducing new distributions called fracture functions (measuring the probabilities of finding a parton and a hadron in the target).

Moreover, it has also been shown that, at NLO, fracture functions are essential in order to factorize collinear singularities related to the emission of partons in the target direction, in processes where the polarization is either neglected [9] or taken into account $[10,11]$. 
Fracture functions are expected to give the dominant contribution to cross sections for the production of leading hadrons in the target fragmentation region. In a given kinematical range, these distributions can be related to the parton distributions of the object exchanged between the initial and final states (a Pomeron in $e p \rightarrow e p X$ or a $\pi^{+}$in $e p \rightarrow e n X$, for example); therefore, their measurement can be used in order to obtain structure functions of "virtual targets," which are beyond the possibilities in a "real target" experiment. It is worth noting that fracture functions allow the most appropriate way to factorize the hadronization properties of the forward direction into nonperturbative distributions and treat them in the context of QCD, whereas other factorization approaches represent effective pictures only valid in a restricted kinematical region.

In that sense, since fracture functions obey evolution equations that differ from the Altarelli-Parisi ones by an inhomogeneous term proportional to the well-known structure and fragmentation functions, their measurement can be taken as a test of new aspects of QCD and, at the same time, can also probe the range of applicability of the cruder factorization approaches. Finally, since both the experimental and theoretical branches of knowledge have been improved during the last years, it seems that a program similar to one developed for structure functions can be applied to fracture functions with, hopefully, a similar success.

Aiming in that direction, we analyze here the most recent experimental results for the production of neutrons in the forward direction in DIS, in the framework of fracture functions, modeling the input fracture distributions exploiting pion exchange ideas, as proposed in Ref. [12], and extend that analysis to the production of pions in the target fragmentation region. This last process is expected to show a clearly different evolution behavior with respect to that driven by the usual evolution equations. The measurement could also be useful in order to test the nonperturbative flux proposed for pions and neutrons in protons.

This paper is organized as follows. In the next section we briefly outline the main features of fracture functions and use the model of Ref. [12], translated into fracture function language for the description of the fragmentation process, to analyze the data. In Sec. III we propose the measurement of forward pions and we estimate the contribution from fracture functions and from the background composed of very forward current fragmentation events. We also analyze there the evolution of these fracture functions and discuss the possibility of finding deviations from the usual evolution. In Sec. IV we summarize our results and present our conclusions.

\section{PERTURBATIVE TREATMENT OF TARGET FRAGMENTATION IN THE VERY FORWARD REGION, MODEL ESTIMATES, AND THEIR COMPARISON WITH THE AVAILABLE DATA}

In the quark-parton model, the semi-inclusive cross section for the production of a hadron $h$ from the deep inelastic scattering of charged leptons carrying momentum $l$ off nucleons of momentum $P$ is usually described in terms of the variables [13]

$$
\begin{gathered}
x=\frac{Q^{2}}{2 P \cdot q}, \quad y=\frac{P \cdot q}{P \cdot l}, \\
z_{h}=\frac{P \cdot h}{p \cdot q}=\frac{E_{h}}{E_{p}(1-x)} \frac{1-\cos \theta_{h}}{2},
\end{gathered}
$$

where $q$ is the transferred momentum $\left(-q^{2}=Q^{2}\right)$ and $E_{h}$, $E_{p}$, and $\theta_{h}$ are the produced hadron and target nucleon energies, and the angle between the hadron and the target in the center of mass of the virtual photon-proton system, respectively. Then, the most naive expression for the unpolarized cross section is

$$
\frac{d \sigma_{p}^{h}}{d x d y d z_{h}}=\frac{1+(1-y)^{2}}{2 y^{2}} \sum_{i=q, \bar{q}} c_{i} f_{i / p}(x) D_{h / i}\left(z_{h}\right),
$$

where $c_{i}=4 \pi e_{q_{i}}^{2} \alpha^{2} / x(P+l)^{2}, f_{i / p}$ is the parton distribution of flavor $i$, and $D_{h / i}$ is the fragmentation function of a hadron $h$ from a parton $i$. Next-to-leading order corrections to this cross section are also known, and have been shown to give a very good description of data with $\theta_{h}>\pi / 2$ [14], the socalled current fragmentation region. However, the target fragmentation region, which corresponds to $\theta_{h}=0\left(z_{h}=0\right)$, cannot be described with the simplified picture of Eq. (2). First of all, it is easy to see that, at the lowest order, hadrons can only be produced antiparallel to the target nucleon $\left(\theta_{h}=\pi\right)$, excluding the forward configurations. On the other hand, going to next-to-leading order, the corrections to the cross section (more precisely, the next-to-leading order coefficients) develop terms proportional to $1 / z_{h}$. This divergence is not only related to soft emission $\left(E_{h}=0\right)$, but also to collinear configurations where hadrons are produced in the direction of the remnant target $\left(\theta_{h}=0\right)$. In this way, since at lowest order hadrons cannot be produced in that direction, it is not possible to factorize the divergence, with the usual procedure, into parton distributions and fragmentation functions.

Then, in order to describe hadrons produced in the target fragmentation region at the lowest order, and also to be able to perform at higher orders a consistent factorization of divergences originated in the current fragmentation region (when a parton is emitted collinearly with the target), a new distribution has to be introduced, the so-called fracture functions $M_{i, h / N}(x,(1-x) z)[8,9]$. These distributions represent the probability of finding a parton of flavor $i$ and a hadron $h$ in the target $N$. It has also been shown that, within this picture, it is convenient to introduce a new variable $z=E_{h} / E_{p}(1-x)$, which allows a discrimination between the $\theta_{h}=0$ and the $E_{h}=0$ configurations, both leading to divergences but of a different nature. The variable $z$ is equal to zero only in the case of soft hadron emission [9].

Therefore the leading order expression for the cross section becomes

$$
\begin{aligned}
\frac{d \sigma_{p}^{h}}{d x d y d z}= & \frac{1+(1-y)^{2}}{2 y^{2}} \sum_{i=q, \bar{q}} c_{i}\left[f_{i / p}(x) D_{h / i}(z)\right. \\
& \left.+(1-x) M_{i, h / p}(x,(1-x) z)\right] .
\end{aligned}
$$

In the next-to-leading order, the corrections to the four cross sections that can be defined taking into account the polarizations of the initial and final state $1 / 2$ spin hadrons have been computed and can be found in Refs. [9-11]. 
The scale dependence of fracture functions at $O\left(\alpha_{s}\right)$ is driven by two kinds of processes, which contribute to the production of hadrons in the remnant target direction: the emission of collinear partons from those found in the target (the usual source of scale dependence of parton distributions, often called homogeneous evolution) and those where partons radiated from the one, to be struck by the virtual probe, fragment into the measured hadron (the so-called inhomogeneous term). These two contributions lead at leading order to the equation

$$
\begin{aligned}
& \frac{\partial}{\partial \ln Q^{2}} M_{i, h / p}\left(\xi, \zeta, Q^{2}\right) \\
& =\frac{\alpha_{s}\left(Q^{2}\right)}{2 \pi} \int_{\xi /(1-\zeta)}^{1} \frac{d u}{u} P_{j}^{i}(u) M_{j, h / N}\left(\frac{\xi}{u}, \zeta, Q^{2}\right) \\
& \quad+\frac{\alpha_{s}\left(Q^{2}\right)}{2 \pi} \int_{\xi}^{\xi /(\xi+\zeta)} \frac{d u}{\xi(1-u)} \hat{P}_{j}^{i, l}(u) f_{j / p}\left(\frac{\xi}{u}, Q^{2}\right) \\
& \quad \times D_{h / l}\left(\frac{\zeta u}{\xi(1-u)}, Q^{2}\right),
\end{aligned}
$$

where $P_{j}^{i}(u)$ and $\hat{P}_{j}^{i, l}(u)$ are the regularized [1] and real [15] Altarelli-Parisi splitting functions, respectively.

As was mentioned in the Introduction, the ZEUS Collaboration has measured DIS events identifying high-energy neutrons in the final state, at very small angles with respect to the proton direction $\left(\theta_{\mathrm{lab}} \leqslant 0.75 \mathrm{mrad}\right)$, in the kinematical range given by $3 \times 10^{-4}<x<6 \times 10^{-3}, \quad 10<Q^{2}<100$ $\mathrm{GeV}^{2}$, and high $x_{L} \equiv \widetilde{E_{n}} / \widetilde{E_{p}}>0.30$. Here $\widetilde{E_{p}}$ and $\widetilde{E_{n}}$ are the target proton energy and that of the produced hadron, but in the laboratory frame, which are straightforwardly related to $E_{h}$ and $E_{p}$ by the appropriate boost. The variables $x_{L}$, $\theta_{\text {lab }}$, and $z$ are then correlated specifically for $\theta_{\text {lab }} \simeq 0$, $x_{L} \simeq z(1-x)$.

The ZEUS Collaboration have reported that events with $x_{L} \geqslant 0.50$ represent a substantial fraction (of the order of $10 \%$ ) of DIS events, which means a contribution comparable in magnitude to the one given by the longitudinal structure function $F_{L}$.

In the framework of a picture for semi-inclusive processes including fracture functions, as the one outlined above, the ZEUS findings can be represented by

$\frac{\int_{0.50}^{1-x}\left(d \sigma_{p}^{h} / d x d y d x_{L}\right) d x_{L}}{d \sigma_{p} / d x d y} \equiv \frac{\int_{0.50}^{1-x} M_{2}^{n / p}\left(x, x_{L}, Q^{2}\right) d x_{L}}{F_{2}^{p}\left(x, Q^{2}\right)}$,

an expression that is exact at leading order due to the fact that, as was mentioned earlier, at this order there is no contribution from currrent fragmentation processes to the target region. In Eq. (5) we have also defined the equivalent to $F_{2}$ for fracture functions,

$$
M_{2}^{n / p}\left(x, x_{L}, Q^{2}\right) \equiv x \sum_{i} e_{i}^{2} M_{i, n / p}\left(x, x_{L}, Q^{2}\right),
$$

and we have made explicit the integration over a finite (measured) range of $x_{L}$. Notice also that if the requirement $\theta_{\mathrm{lab}} \simeq 0$ is not satisfied, the exact relation between $x_{L}$ and $z$ has to be taken into account.

In Fig. 1 we show the experimental outcome for this frac-

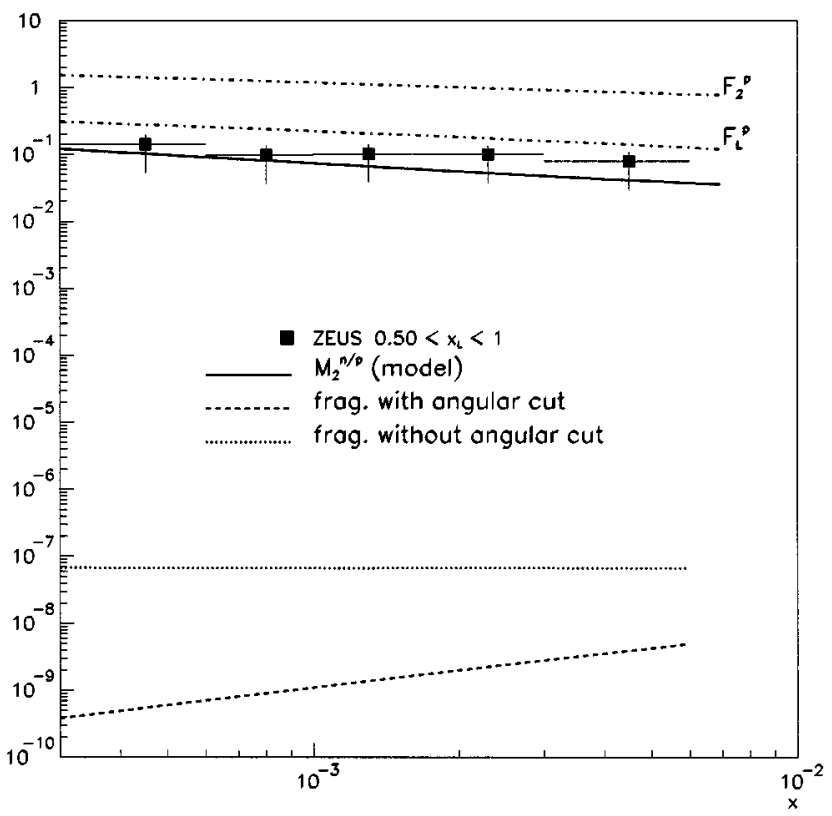

FIG. 1. Fracture function of neutrons in protons as measured by ZEUS compared with the model prediction and current fragmentation contributions.

ture function [as defined in Eq. (5) and at $Q^{2}=10 \mathrm{GeV}^{2}$, taking advantage of the negligible $Q^{2}$ dependence of the data], and we compare it to $F_{2}^{p}$ and $F_{L}^{p}$. We also show the contribution to the same observable coming from current fragmentation processes, computed taking into account the appropriate boost to the laboratory system and the cut in the $\theta_{\text {lab }}$ angle implemented in the experimental determination. It is worth noting that this last contribution is pure NLO and in fact it is about eight orders of magnitude smaller than the experimental data. ${ }^{1}$ Furthermore, as can be seen in the figure, even releasing the constraint on the angle for the final state neutron (but keeping the requirement of energy fraction $>0.50$ ) the contribution still remains negligible, basically because of the kinematical suppression (the effect of the boost from the $\gamma^{*} p$ center-of-mass system to the laboratory frame) and to the fact that this contribution begins at NLO.

Fracture functions, as parton distributions in general, are essentially of a nonperturbative nature and have to be extracted from experiment. However, their close relation with fragmentation and structure functions allows in certain extreme cases a model estimate for them. Basically, the task amounts to modeling the target fragmentation process and then writing down the result in the language of fracture functions. This procedure provides input fracture functions, or at least hints of their functional shape, at a definite scale, which can then be compared with actual measurements and evolved with the corresponding evolution equations.

Recently, a very sensible model estimate for the production of forward hadrons in DIS has been proposed ${ }^{2}$ [12], exploiting the idea of nonperturbative Fock components of the nucleon. In this approach the semi-inclusive DIS cross

\footnotetext{
${ }^{1}$ For our analysis, we use distributions from Refs. [16,17].

${ }^{2}$ In Ref. [18] an approach for the residual effects of fracture functions in the currrent fragmentation region has also been proposed.
} 




FIG. 2. The $x_{L}$ dependence of the model compared with the ZEUS data, integrated in the region $3 \times 10^{-4}<x<6 \times 10^{-3}$.

sections, and through them the corresponding fracture functions at a certain input scale $Q_{0}^{2}$, can be interpreted as the product of a flux of neutrons in the proton (integrated over $p_{T}^{2}$ ) times the structure function of the pion exchanged between them; i.e,

$$
M_{2}^{n / p}\left(x, x_{L}, Q_{0}^{2}\right) \simeq \phi_{n / p}\left(x_{L}\right) F_{2}^{\pi^{+}}\left(\frac{x}{1-x_{L}}, Q_{0}^{2}\right)
$$

Actually, Ref. [12] is a proposal to measure the structure function of pions at very small $x$ using a nonperturbative computation of the flux, which is in very good agreement with experimental data on high-energy neutron and $\Delta^{++}$ production in hadron-hadron collisions [19]. The structure functions of pions have been measured with some precision only for $x>0.10$, but there are parametrizations based on dynamical parton model assumptions (valencelike distributions at low $\mu_{0}^{2}$ ) [20], which have shown an impressive predictive power at small $x$ for the proton structure function [16], and allow us to make a rough comparison between the model estimate and the data. In Fig. 1 we show the that model estimate, taking $Q_{0}^{2}=10 \mathrm{GeV}^{2}$, is in excellent agreement with the data. In Fig. 2 we also compare the $x_{L}$ dependence of the data and of the model prediction (normalized to the same number of events in the measured interval), showing also a good agreement. Regarding the shift in the position of the peak, it is worth noticing that the abovementioned ZEUS data have not been corrected either for finite acceptance effects or for those related with finite resolution, and the former may account for some, perhaps all, of the observed shift to largers values of $x_{L}$ [21]. This would eventually give an even better agreement between the model and the data.

\section{SCALE DEPENDENCE OF FRACTURE FUNCTIONS: PIONS IN THE FINAL STATE}

The success of the model estimate encourages us to go further and use the functional dependence of fracture functions, induced by the model and corroborated by the data, to analyze also the $Q^{2}$ dependence.

We first analyze the more familar process of neutron production. Since the probability of current parton fragmentation into a neutron (given by fragmentation functions) is comparatively small with respect to that of processes originated in the target (fracture functions), no significant effects are expected in the scale evolution arising from the inhomogeneous term in this process. The evolution is mainly driven by the usual homogeneous term of the evolution equations, leading to an almost constant ratio between the number of neutron tagged events and that of all DIS events, as observed by ZEUS.

However, the scale dependence induced in the cross section for the production of pions, at least in the kinematical region of very small $x$ and small $x_{L}$, can be considerably affected by the inhomogeneity, given that soft pions are produced more copiously from quarks than neutrons. ${ }^{3}$ Of course, in order to analyze these features of the evolution, an estimate for the proton to pion fracture function at some input scale $Q_{0}^{2}$ is required. For this purpose, we can use the same ideas formerly applied to neutron production, noticing two further advantages. First, the flux to be used can be straightforwardly obtained from the one used in the last section by means of the crossing relation

$$
\phi_{\pi^{+} / p}\left(x_{L}\right)=\phi_{n / p}^{\pi^{+}}\left(1-x_{L}\right)
$$

where the superscript $\pi^{+}$indicates that the relation is valid only when the contribution due to the exchange of positively charged pions is considered. The second advantage is related to fact that the neutron structure function is much better known than that for pions. Taking into account these features and neglecting the insignificant contributions coming from $\Delta$ exchange, the proton-to-pion fracture function can be approximated by

$$
M_{2}^{\pi^{+} / p}\left(x, x_{L}, Q_{0}^{2}\right) \simeq \phi_{\pi^{+} / p}\left(x_{L}\right) F_{2}^{n}\left(\frac{x}{1-x_{L}}, Q_{0}^{2}\right) .
$$

Additionally, the direct measurement of this fracture function could be of interest as a double test of the crude factorization ideas.

Notice that an analogous reasoning can be made considering the exchange and production of $\pi^{0}$ mesons, but with a proton instead of a neutron as the final state and intermediate particle, respectively. The study of leading $\pi^{0}$ production may also be possible at ZEUS [21].

In Fig. 3 we show the model estimates for proton-to-pion fracture functions (taking $Q_{0}^{2}=4 \mathrm{GeV}^{2}$ ), integrated over two different bins of $x_{L}$, compared with the contribution coming from the current fragmentation processes. We assume here the same restrictions as in the data from the ZEUS Collabo-

\footnotetext{
${ }^{3} \mathrm{We}$ are indebted to G. Veneziano for calling our attention to this point.
} 


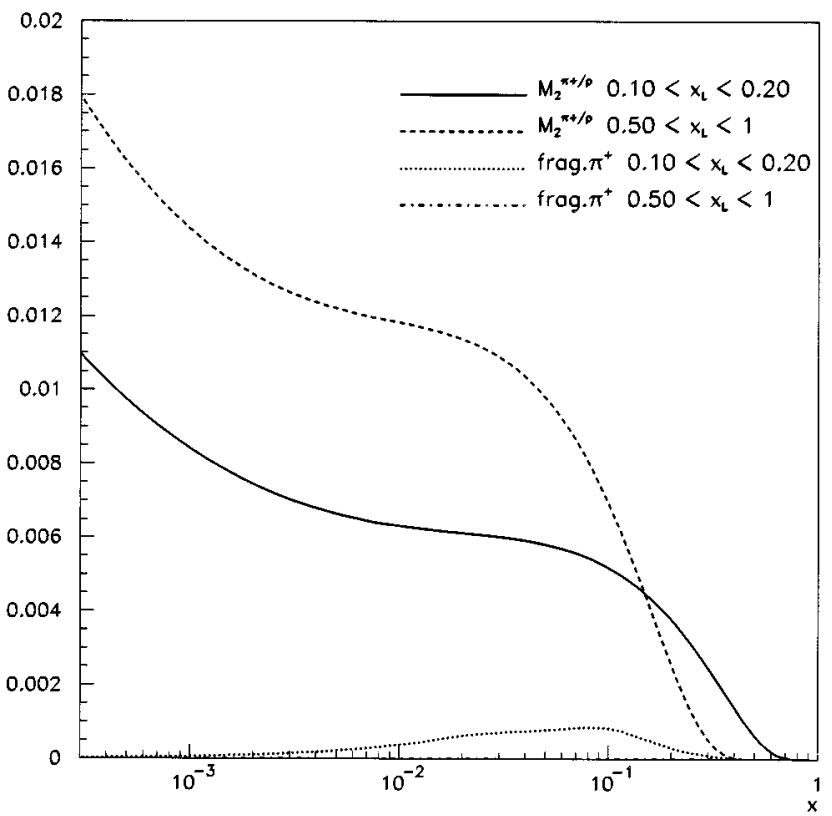

FIG. 3. Prediction for the fracture function of $\pi^{+}$in protons for two different bins of $x_{L}$ and the current fragmentation contribution.

ration for neutron production. ${ }^{4}$ As can be seen, higher-order fragmentation contributions are quite less significant than fracture contributions, even at the lowest $x_{L}$ bin.

Of course, the model is not expected to work over the whole kinematical region and, in fact, any deviation from the scale dependence implied in Eq. (9) (note that the flux is

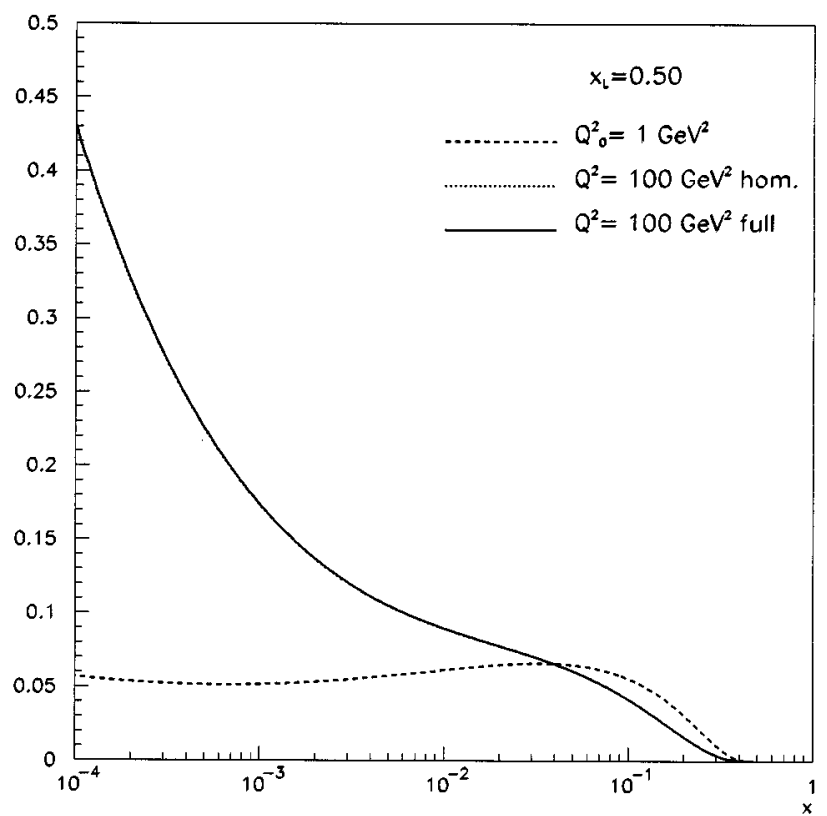

FIG. 4. Evolution of the fracture function of $\pi^{+}$in protons for $x_{L}=0.50$ and $Q_{0}^{2}=1 \mathrm{GeV}^{2}$.

\footnotetext{
${ }^{4}$ Actually, it would also be interesting to count with the $p_{T}$ distribution of forward hadrons in order to establish the exact range where fracture function contribution dominates.
}

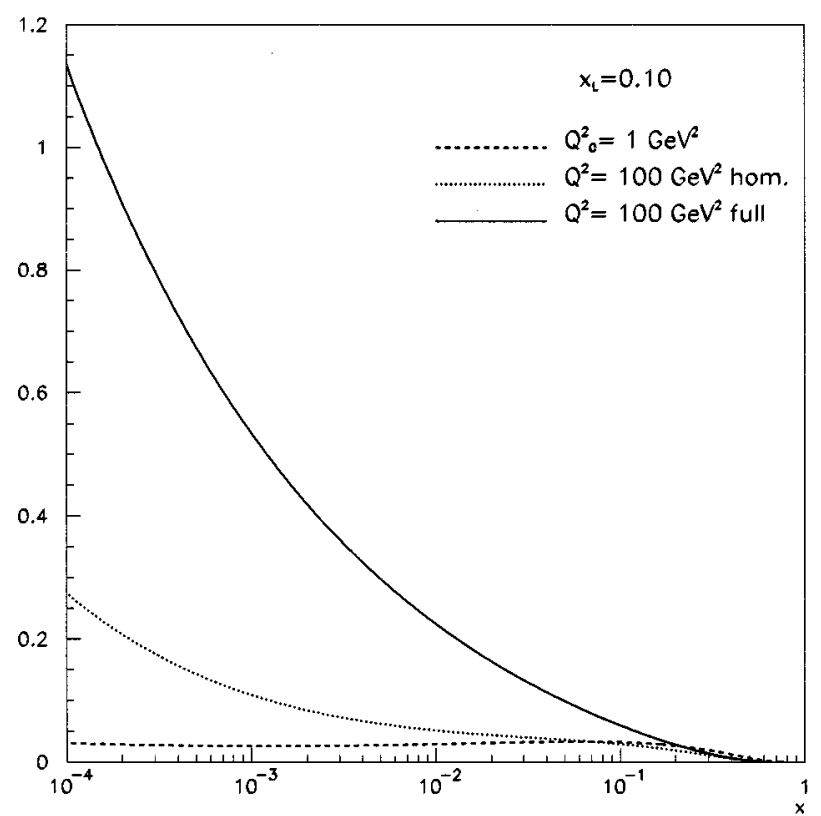

FIG. 5. The same as in Fig. 4 with $x_{L}=0.10$.

assumed to be $Q^{2}$ independent) would show the breakdown of the approximated factorization hypothesis. However, the Ansatz in Eq. (9) can be taken as an effective relation, valid at some initial value of $Q_{0}^{2}$, for which the estimated flux is adequate, and therefore provides a sensible input distribution. ${ }^{5}$ As usual, the correct scale dependence is that given by the evolution equations for fracture functions, and that is the aim of our next step.

In order to study the effect of the inhomogeneity in the

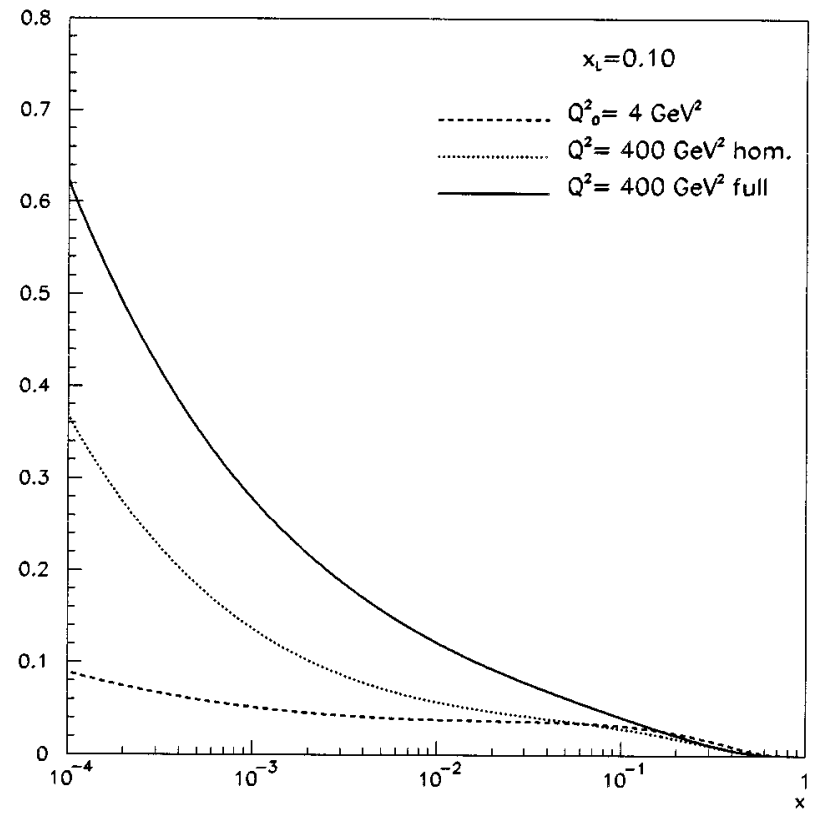

FIG. 6. The same as in Fig. 5 with $Q_{0}^{2}=4 \mathrm{GeV}^{2}$.

${ }^{5}$ In fact, different choices of $Q_{0}^{2}$ do not modify the overall trends of the evolution. 


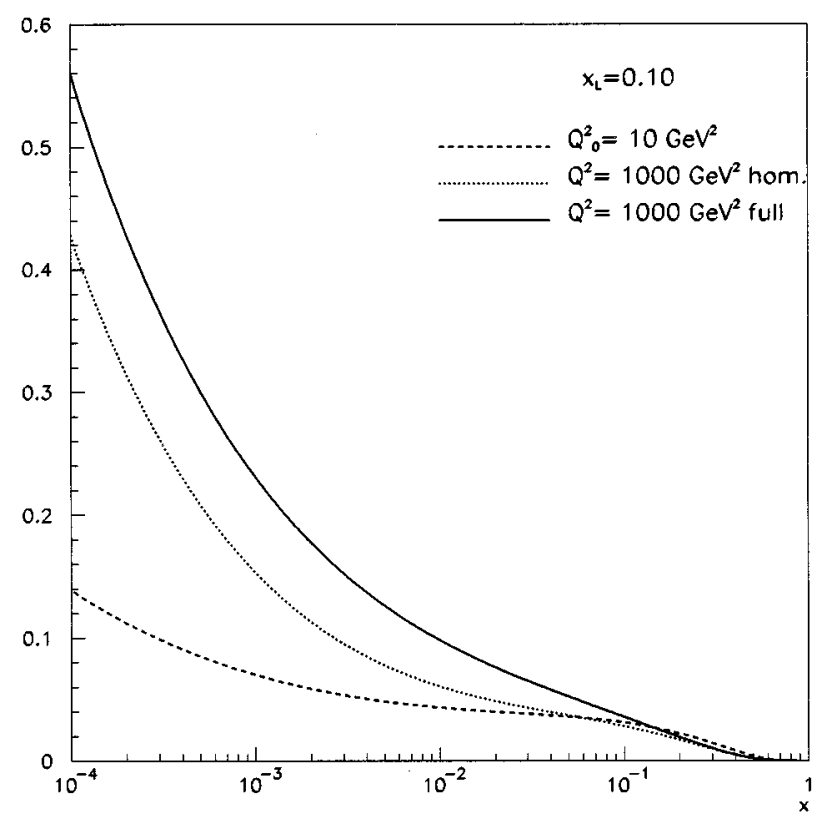

FIG. 7. The same as in Fig. 5 with $Q_{0}^{2}=10 \mathrm{GeV}^{2}$.

evolution we take different values of $x_{L}$, and keep them fixed while we analyze the $x$ and $Q^{2}$ dependence of fracture functions induced by both the homogeneous and the complete evolution equations.

In Fig. 4 we show the result of an evolution from $Q_{0}^{2}=1 \mathrm{GeV}^{2}$ at $x_{L}=0.50$. Both solutions (the homogeneous and the complete) are superimposed, the difference being less than $0.1 \%$. These behaviors are perfectly compatible with the results obtained by the ZEUS Collaboration in the case of neutron production (where the inhomogeneity contributes about 10 times less) in the same kinematical region, where no difference has been found in the evolution between $F_{2}$ and $M_{2}$.

However, for smaller $x_{L}$ the situation is completely different. As the fragmentation function increases with lower values of the argument, the inhomogeneous contribution becomes much more relevant and its effect in the evolution is sizable. In fact, Fig. 5 shows the evolution result for $x_{L}=0.10$ and $Q_{0}^{2}=1 \mathrm{GeV}^{2}$, where the full evolution results outsize the homogeneous one by a factor of 4 at small $x$. These corrections are smaller if the Ansatz of Eq. (9) is assumed to be valid at values of $Q_{0}^{2}=4 \mathrm{GeV}^{2}$ (Fig. 6) and $Q_{0}^{2}=10 \mathrm{GeV}^{2}$ (Fig. 7), but still remain considerable and show the same behavior.

The uncertainty due to the estimate of the input fracture functions at some initial scale obviously will be lifted as soon as they are measured; it will be then possible to study their evolution in a model-independent way, completely determined by QCD, and to compare the result with the experimental data at different values of the scale.

In Fig. 8, we show for completeness the results obtained using a bin in $x_{L}$ between 0.10 and 0.20 . In that case the weight of the corrections is lowered by the inclusion of larger- $x_{L}$ terms, but it is noticeable that they are still about $20 \%$, whereas corrections from the fragmentation processes to the fracture function, as in Fig. 3, are less than $1 \%$ in the small- $x$ range. This is due to the kinematical restrictions im-

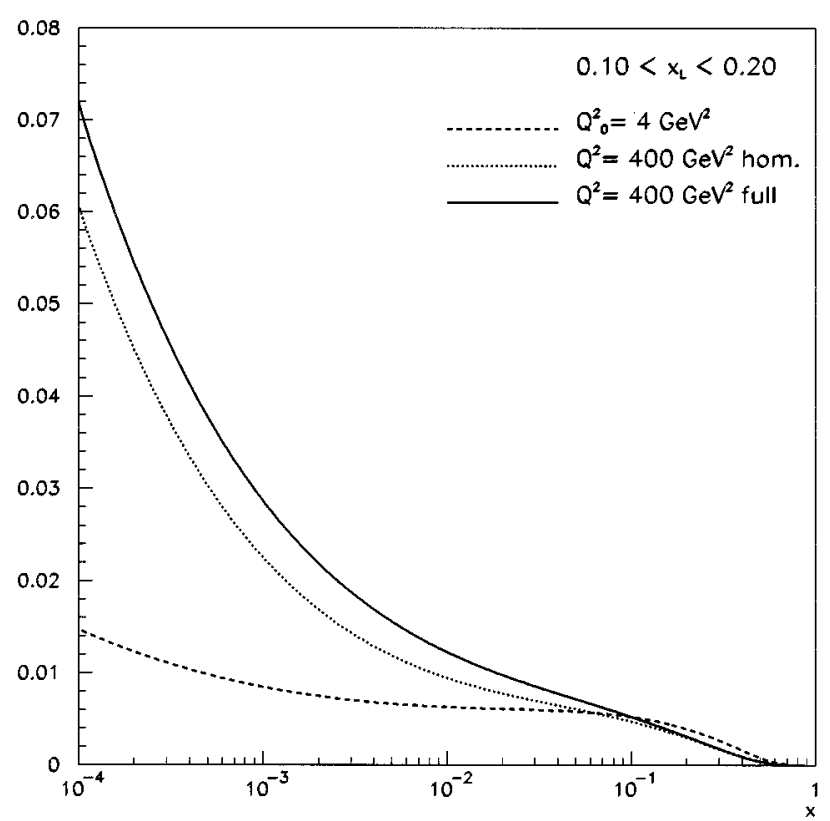

FIG. 8. The same as in Fig. 6 but integrating over a bin of $x_{L}$.

posed over the final state, but also because to the fact that the fragmentation contribution appears at the same order of $\alpha_{s}$ in the evolution, but one order higher in the cross section than that coming from the fracture one.

\section{SUMMARY AND CONCLUSIONS}

In this paper, we have analyzed recent experimental data on the production of forward neutrons in DIS in terms of fracture functions, finding that the main features of the data can be fairly reproduced by this perturbative QCD approach, once a nonperturbative model estimate for the input fracture functions is given. Studying the evolution properties of these fracture functions in the specific case of forward pions in the final state, we have found that the effects of the inhomogeneous term in the evolution equations are large and measurable, particularly in the kinematical region of very small $x$ (accessible to HERA) and small $x_{L}$. These effects are negligible for large values of $x_{L}$, justifying the use of the usual homogeneous Altarelli-Parisi equations for, as an example, the $t$-integrated diffractive structure function $F_{2}^{D(3)}\left(x_{\mathrm{P}}, \beta, Q^{2}\right)$, which is just the fracture function of protons in protons $M_{2}^{p / p}\left(\beta x_{\mathrm{p}},\left(1-x_{\mathrm{P}}\right), Q^{2}\right)[22,23]$.

The measurement of the production of pions in the target fragmentation region in DIS will be useful to check the range of applicability of factorization Ansätze for fragmentation processes and to perform a new test of QCD by means of the analysis of the evolution of fracture functions, which is predicted to be different from the usual one in the kinematical region alluded to before.

\section{ACKNOWLEDGMENTS}

We gratefully acknowledge C. García Canal, G. Veneziano, and G. Levman for enlightening discussions and for carefully reading the manuscript. 
[1] G. Altarelli and G. Parisi, Nucl. Phys. B126, 298 (1977).

[2] R. D. Ball and S. Forte, Phys. Lett. B 335, 77 (1994).

[3] J. Ellis and R. L. Jaffe, Phys. Rev. D 9, 1444, (1974); 10, 1669 (1974).

[4] G. Altarelli and G. G. Ross, Phys. Lett. B 212, 391 (1988).

[5] ZEUS Collaboration, M. Derrick et al., Phys. Lett. B 315, 481 (1993); ZEUS Collaboration, M. Derrick et al., ibid. 332, 228 (1994); ZEUS Collaboration, M. Derrick et al., ibid. 338, 483 (1994); H1 Collaboration, T. Ahmed et al., Nucl. Phys. B429, 477 (1994).

[6] ZEUS Collaboration, M. Derrick et al., in Proceedings of the XVIII International Conference on High Energy Physics, Warsaw, 1996 (unpublished).

[7] ZEUS Collaboration, M. Derrick et al., Phys. Lett. B 384, 388 (1996).

[8] L. Trentadue and G. Veneziano, Phys. Lett. B 323, 201 (1994).

[9] D. Graudenz, Nucl. Phys. B432, 351 (1994).

[10] D. de Florian, C. A. García Canal, and R. Sassot, Nucl. Phys. B470, 195 (1996).
[11] D. de Florian and R. Sassot, Nucl. Phys. B488, 367 (1997).

[12] H. Holtmann et al., Phys. Lett. B 338, 363 (1994).

[13] G. Altarelli, R. K. Ellis, G. Martinelli, and S. Y. Pi, Nucl. Phys. B160, 301 (1979).

[14] D. Graudenz, Report No. CERN-TH/96-155, hep-ph/9606470 1996 (unpublished).

[15] E. Konishi, A. Ukawa, and G. Veneziano, Nucl. Phys. B157, 45 (1979).

[16] M. Glück, E. Reya, and A. Vogt, Z. Phys. C 67, 433 (1995).

[17] J. Binnewies, B. A. Kniehl, and G. Kramer, Phys. Rev. D 52, 4947 (1995).

[18] D. de Florian et al., Phys. Lett. B 389, 358 (1996).

[19] V. R. Zoller, Z. Phys. C 53, 443 (1992).

[20] M. Glück, E. Reya, and A. Vogt, Z. Phys. C 53, 651 (1992).

[21] G. Levman (private communication).

[22] D. Graudenz and G. Veneziano, Phys. Lett. B 365, 302 (1996).

[23] Z. Kunszt and W. J. Stirling, Report No. hep-ph/9609245, 1996 (unpublished). 